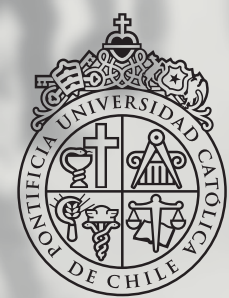

PONTIFICIA

UNIVERSIDAD

CATÓLICA

DE CHILE
Revista

Trabajo
Análisis crítico

de la teoría de cambio en intervenciones de visitas domiciliarias

en el programa

Chile Crece Contigo

Kenichi Haramoto

Paula Verdugo

Visita domiciliaria en Trabajo Social:

¿cómo la valoran y utilizan un grupo de estudiantes de la profesión?

Paula Chamorro

Alicia Razeto
Propuesta de un modelo de supervisión reflexiva de visitas domiciliarias realizadas en un programa dirigido a familias de recién nacidos

María Carolina Velasco Hodgson

Daniela Tuma IBIETA

Claudia Aldana Pereira

\section{Formación continua en} visita domiciliaria.

Análisis cualitativo de una metasupervisión reflexiva

Claudia Cerfogli

Paulina Contreras

Experiencias sobre el uso de eHealth en educación para la salud, gestación y primera infancia:

una revisión bibliográfica

Benjamin Charles Nielsen Guzmán 



\title{
Visita domiciliaria en Trabajo Social: ¿cómo la valoran y utilizan un grupo de estudiantes de la profesión? .'2 $^{12}$
}

\section{Home visit in Social Work: how a group of students value and use it?}

\section{Paula Chamorro}

Estudiante de Trabajo Social de la Pontificia Universidad Católica de Chile.Correo electrónico: pachamorro@uc.cl

\section{Alicia Razeto}

Académica Escuela de Trabajo Social, Pontificia Universidad Católica de Chile. Doctora en Ciencias de la Educación. Av. Vicuña Mackenna 4860, Macul, Santiago.Correo electrónico: arazeto@uc.cl

\begin{abstract}
Resumen
Pese a ser una de las estrategias de intervención emblemáticas del Trabajo Social, los estudios nacionales publicados sobre la visita domiciliaria se han realizado mayoritariamente desde el campo de la salud y la psicología. En este contexto, el articulo muestra los resultados de un estudio que tuvo como objetivo general analizar cómo utilizan la estrategia de visita domiciliaria los estudiantes de Trabajo Social que realizan o han realizado algún semestre de práctica profesional y qué opinión tienen dichos estudiantes sobre las cualidades y dificultades de la visita domiciliaria como estrategia de intervención social. El método del estudio contempló la aplicación de dos técnicas de recolección de información: i) una encuesta a una muestra total de 51 estudiantes de cuarto y quinto año de la carrera de Trabajo Social de una universidad, ii) un grupo de discusión con 10 estudiantes de cuarto y quinto año de la carrera de Trabajo Social.

Como principales resultados se pudo constatar que, en este grupo de estudiantes de esta universidad, la estrategia es utilizada y bien valorada por sus aportes. Sin embargo, se detectó que principalmente la utilizan para propósitos de recolección de información sobre el caso o familia intervenida y no como estrategia para entregar servicios sociales o educativos. También se encontraron ciertas distancias entre el uso dado por los estudiantes y lo recomendado por la literatura revisada, en relación con la frecuencia, intensidad, instrumentos y preparación profesional en el uso de la visita.
\end{abstract}

Palabras clave: Visita domiciliaria, estudiantes universitarios, Trabajo Social

\begin{abstract}
In spite of being one of the emblematic strategies of intervention of the Social Work, the national studies published on the home visit have been realized for the most part from the field of the health and the psychology. In this context, this paper is the product of a field study it had as general aim analyze how there use the strategy of domiciliary visit the students of Social Work that they realize or have realized some semester of professional practice and what opinion the above mentioned students have on the qualities and difficulties of the domiciliary visit as strategy of social intervention. The method of the study contemplated the application of two technologies of compilation of information: i) a survey to a total sample of 51 students of fourth and fifth year of the career of Social Work of a university, ii) a group of discussion with 10 students of fourth and fifth year of the career of Social Work.

Since principal results it was possible to state that in this group of students of this university, the strategy is used and valued well by his contributions. Nevertheless, there was detected that principally they use for intentions of compilation of information about the case or controlled family and not as strategy to deliver social or educational services. Also they found certain distances between the use given by the students and the recommended for the checked literature, in relation to the frequency, intensity, instruments and professional preparation in the use of the visit.

Key words: Home visit, university students, Social Work
\end{abstract}




\section{Introducción}

En Estados Unidos, y más recientemente en Europa, los programas de visita domiciliaria son cada vez más populares y son utilizados como intervención temprana en infancia ${ }^{3}$. Según un estudio realizado por Saias, Lerner, Greacen, Simon-Vernier \& Emer (2012), este tipo de programas sirven a más de 500.00 familias en los Estados Unidos y ha ido ganando interés en Europa como parte del sistema de prevención que compone el estado de bienestar. En Chile, desde el momento de fundación de la profesión del Trabajo Social, durante la primera mitad del siglo $\mathrm{XX}^{4}$, las entonces denominadas visitadoras sociales ${ }^{5}$ comienzan a utilizar la visita domiciliaria como estrategia fundamental para conocer el medio en el que se desarrolla el niño y su familia, el ambiente familiar, e investigar las causas de los problemas que viven, para así solucionar dichas problemáticas y ofrecer protección al hogar (Zamorano, 1935; López, 1949; y Tardel, 1947). De ahí en adelante, las visitas domiciliarias forman parte del quehacer habitual de los trabajadores sociales en los distintos contextos sociopolíticos. El año 2002, con la creación del Chile Solidario y el Programa Puente, los apoyos familiares utilizan la visita domiciliaria como estrategia central para brindar el acompañamiento a las familias de extrema pobreza. En salud, a partir de 1998 con la reforma de los consultorios a los centros de salud familiar, la visita domiciliaria adopta importancia al ser una estrategia consonante con el modelo de atención integral, familiar y comunitario de estos centros (Ministerio de Salud, 2008). Más tarde, el año 2006, la visita domiciliaria se reconoce como una estrategia prioritaria de acción desde la política pública en el área de salud, a través de la creación de la política de Protección Integral a la Infancia del Sistema Chile Crece Contigo. Implementada principalmente por trabajadores sociales, enfermeros y matrones, las visitas domiciliarias se utilizan desde esta política como "una estrategia de entrega de servicios de salud realizada en el domicilio, dirigida a familias desde la gestación hasta los seis años del niño o niña, basada en la construcción de una relación de ayuda entre el equipo de salud interdisciplinario y las madres, padres o cuidadores primarios, cuyo objetivo principal es la promoción de mejores condiciones ambientales y relacionales para favorecer el desarrollo integral de la infancia" (Chile Crece Contigo, 2009).

El presente artículo se sitúa desde la premisa que la visita domiciliaria como estrategia de intervención social posee un valor estratégico para el Trabajo Social (Tonon, 2005) y que sus posibilidades van más lejos que la simple descripción de los espacios visitados (González, 2014). En consecuencia, el artículo postula que es fundamental posicionar la visita domiciliaria como contenido en la formación de los trabajadores sociales desde la enseñanza universitaria, puesto que su aplicación exige un saber profesional al suponer el manejo de marcos teóricos referenciales y la aplicación de procedimientos científicos. Además, es un acto profesional que tiene un propósito y se realiza bajo los principios éticos de la profesión (González, 2014).

El artículo aquí presentado es resultado de un estudio realizado el año 2015 que buscó describir cómo utilizan la visita domiciliaria estudiantes de Trabajo Social que han realizado al menos un proceso de práctica profesional ${ }^{6}$ en el transcurso de su formación universitaria y qué opinión tienen dichos estudiantes sobre las cualidades y dificultades de la visita domiciliaria como estrategia de intervención social. La pregunta guía de la investigación fue ¿cómo conciben y utilizan la estrategia de visita domiciliaria los estudiantes de Trabajo Social? Se asume que las experiencias preprofesionales con la

1 Este artículo es un producto académico del Fondecyt de Iniciación $N^{\circ} 11140679$ actualmente en curso, titulado "La visita domiciliaria como estrategia de intervención social para aumentar la participación de los padres en la educación de sus hijos en escuelas básicas municipales", cuya investigadora principal es Alicia Razeto.

2 Las autoras agradecen el apoyo en la implementación del estudio que dio origen a este artículo a la trabajadora social de la UC Gabriela Fraczinet.

3 Aunque no se reducen solamente al trabajo con población infantil. Por ejemplo, el año 2005, la Agencia de Protección Ambiental (EPA) de los Estados Unidos publica una Guía para la Implementación de Visitas Domiciliarias en el Asma, con el propósito de entregar instrucciones para implementar un programa de visitas domiciliarias que permitan manejar los factores de riesgo presentes en el hogar y disminuir los gatillantes del asma (EPA, 2005).

4 En Chile, el Trabajo Social surge como profesión en 1925 con la creación de la primera escuela de Trabajo Social por el doctor Alejandro del Río. Más tarde, en 1929, se crea la segunda escuela de Trabajo Social, la Escuela Elvira Matte de Cruchaga, dependiente de la Universidad Católica de Chile (Aylwin y Solar, 2011).

5 Este fue el nombre que recibieron las primeras trabajadoras sociales, denominación cambiada oficialmente en 1957 al crearse el Colegio de Asistentes Sociales (Aylwin y Solar, 2011).

6 En este artículo se agrupa bajo la noción de práctica profesional a las dos experiencias de práctica que los estudiantes realizan en distintos niveles de progresión de la carrera durante su proceso de formación universitaria en la institución participante de este estudio. 
visita domiciliaria influyen la forma en la cual posteriormente esos profesionales se relacionaran con dicha estrategia.

\section{Antecedentes y conceptualizaciones acerca de la estrategia de visita domiciliaria}

Pese a ser una de las estrategias de intervención emblemáticas del Trabajo Social, los estudios nacionales publicados sobre los efectos de la visita domiciliaria se han realizado mayoritariamente desde el campo de la salud y la psicología ${ }^{7}$. Por ejemplo, Aracena et al. (2011) concluyó -mediante una investigación experimental al alero de un proyecto de estudio financiado por Fondecyt- que las visitas domiciliarias como estrategias de intervención tienen impacto en el mejoramiento del bienestar biopsicosocial de las madres y sus hijos en un contexto de deprivación (Aracena et al., 2011). Otras investigaciones internacionales en el campo de la salud han encontrado resultados positivos de las visitas domiciliarias en la salud de recién nacidos, niños y familias en riesgo social (Academia Americana de Pediatría, 1998; Avellar \& Supplee, 2013; Kitzman et al., 1997; Baqui et al., 2008; Gogia \& Sachdev, 2010; Cruz y Martte, 2010), embarazadas (Issel et al., 2014), adultos mayores (Brugerolles et al., 2008; Medina et al., 1998) $y$ en el apoyo a los enfermos terminales (AscencioHuertas et al., 2013; Astudillo et al., 2008).

Un reciente estudio realizado por García, McNaughton, Radosevich, Brandt $\&$ Monsen (2013) en Estados Unidos explica que las visitas domiciliarias son aplicadas por enfermeras como parte de la política de salud pública hacia las mujeres migrantes latinoamericanas. Las enfermeras realizan las visitas domiciliarias a madres vulnerables desde el punto de vista de la salud mental o que presentan factores de riesgo como pobreza, ausencia de seguro de salud, ser madres adolescentes o llevar su maternidad en solitario. El estudio aplicado por García et al., (2013) comprobó que los conocimientos, conductas y estado de las mujeres latinas con problemas de salud mental mejoran tras un programa de visitas domiciliarias. Este programa de visitas domiciliarias es diseñado como intervención temprana para entregar conocimientos y habilidades en torno a las prácticas parentales saludables y del cuidado del nuevo hijo hasta sus primeros años de vida.
La frecuencia de la visita domiciliaria es una variable importante al momento de referirse a su eficacia. Si bien se ha estudiado que a mayor frecuencia de visita mayor el efecto, no se ha determinado con exactitud cuál es la cantidad deseable (Nievar, Van Egeren \& Pollard, 2010). Sin embargo, un estudio de Tubach et al. (2012) encontró que al menos tres visitas al mes serían más efectivas que las que hacen dos visitas o menos. Otra de las variables relevantes para la eficacia es el nivel de capacitación de los que hacen la visita domiciliaria, independientemente de si estos son o no profesionales (Tubach et al., 2012). Por otra parte, la variabilidad de la efectividad dependería también de las prácticas de implementación y las dificultades en el compromiso de las familias. Tubach et al. (2012) también menciona que el número de participantes de un programa de visita domiciliaria influye en su efectividad. Cuando el público es muy grande, los recursos para cada familia se ven disminuidos y los modelos pueden no ser replicados exactamente. En este sentido, dice Tubach et al. (2012), hay que tener en cuenta que los estudios piloto aplicados por universidades pueden tener resultados superiores porque se concentran en un número pequeño de participantes; es mejor la calidad de la supervisión de la visita domiciliaria o por cambios en las necesidades y experiencias de madres de bajos recursos a lo largo del tiempo.

Podría definirse que la visita domiciliaria consiste en la entrega de un servicio de ayuda, información o apoyo a un individuo y a su familia (Boller, Strong \& Daro, 2010) que se realiza en el propio hogar a través de encuentros habituales realizados entre la familia y un trabajador capacitado que puede ser o no profesional (Avellar \& Supplee, 2013; Aracena et al., 2011; Wasik \& Bryant, 2001). De acuerdo a Boller et al. (2010) existe una amplia gama de servicios de apoyo que el agente visitador puede entregar a la familia, tales como:

- Fortalecer la relación entre padres e hijos.

- Propiciar el desarrollo de un ambiente más seguro y estimulante para los niños.

- Fomentar el bienestar del niño y su preparación para la escuela.

- Entregar mayores conocimientos a los padres sobre el desarrollo del niño.

- Promover el bienestar de los padres.

7 Sin embargo, es preciso señalar que, si bien los programas de visita domiciliaria han sido usados desde hace décadas en Chile, la sistematización y evaluación de estas experiencias ha sido muy escasa, encontrándose documentada muchas veces en textos de circulación restringida, documentos de trabajo o informes institucionales internos (Kotliarenco et al., 2010). 
Además de entregar un servicio, otros objetivos de la visita pueden ser evaluar y recolectar información acerca de la situación que están viviendo las familias: "las visitas domiciliarias proporcionan al profesional información clave para evaluar los puntos fuertes de la familia en el contexto del entorno físico del barrio y el hogar, las interacciones familiares y los recursos de la familia" (Allen y Tracy, 2004, p. 202). Dentro de este objetivo es preciso reconocer que en la visita domiciliaria suelen aplicarse dos técnicas que facilitan la recolección de información y guían la interacción entre el profesional y el usuario: la observación y la entrevista (Tonon, 2005).

A pesar de ser una estrategia que acompaña la historia del Trabajo Social, la visita domiciliaria puede ser aplicada por diversos profesionales, dado que la ayuda que el agente intenta brindar a la familia puede estar enfocada en necesidades sociales, emocionales, cognitivas, de salud o educativas. En consecuencia, pueden realizarla las enfermeras y los profesores (Wasik \& Bryant, 2001).

Varias son las ventajas que ofrece esta estrategia, siendo la principal que se desarrolla en el escenario fundamental y ambiente natural en donde se llevan a cabo las principales interacciones entre padres e hijos, como lo es el hogar, siendo este último un ambiente particularmente favorable para observar, modelar, enseñar e influir en su interacción (Allen y Tracy, 2004). Eso permite conocer a la familia en sus reales condiciones de vida y observar la interacción familiar directamente. Una de las ventajas más evidentes de los programas de visitas domiciliarias es que se maximiza la probabilidad que la familia participe, pues el servicio se entrega en el hogar, esto es ibeneficioso? especialmente en las familias en riesgo que tienen más dificultades de participar de los programas (Nievar et al., 2010). Como se realiza en el hogar, posibilita conocer a miembros de la familia que no llegan a las instituciones por dificultades de desplazamiento, por sentirse extraños en el ambiente institucional (Aylwin y Solar, 2011) o por la situación de pobreza en la que viven dado que son los que presentan más dificultades para acceder a los sistemas formales (Aracena et al., 2011). En este sentido, las visitas domiciliarias evitan el costo de transporte y el cuidado de los niños que no son el blanco de la intervención (Nievar et al., 2010).

Por otra parte, al realizarla en casa, permite evitar la actitud defensiva que los usuarios pueden presentar en el entorno formal del despacho profesional (Lázaro, 2007), contribuyendo a balancear la relación asimétrica de poder entre el profesional y la familia, ayudando a construir relaciones de confianza (Allen y Tracy, 2004).

Según los propósitos que tengan las visitas domiciliarias, tres son los principales tipos (Masten y Coatsworth, 1998):

- Centradas en el riesgo, las que buscan prevenir y eliminar factores de riesgo o bien mitigar sus efectos luego de ocurrido el problema.

- Centradas en el proceso, en las que se busca fomentar el desarrollo de las características y fortalezas de la familia, mejorando el aprendizaje en el hogar.

- Centradas en las estrategias de recursos, en las que se busca fortalecer las redes de las familias de manera tal de potenciar el acceso a los recursos.

De acuerdo al Ministerio de Salud (2009), en el contexto del sistema Chile Crece Contigo existen cuatro modelos de visita domiciliaria, los que son:

- Basado en el acceso a los servicios: que buscan disminuir los estresores al interior de la familia y apoyar el desarrollo saludable del niño, a través de la vinculación con los recursos y servicios comunitarios requeridos para satisfacer sus necesidades.

- Basado en la entrega de apoyo y educación: que persiguen aumentar los conocimientos y habilidades parentales en tema de cuidado y desarrollo infantil.

- Basado en el comportamiento: que buscan mejorar la relación de los padres con sus hijos a través de la observación y respuesta a los comportamientos del niño.

- Basado en la relación: donde el profesional acompaña al individuo o familia en una situación de vida particular, estableciendo una relación de confianza entre el profesional que realiza la visita y la familia. Requiere en muchos casos el profesional alguna preparación psicoterapéutica.

Los agentes visitadores deben poseer una gama de experiencias y credenciales (Boller et al., 2010) lo que significa que se requiere de cierta experticia para aplicar las visitas domiciliarias. También se requiere de habilidades comunicacionales y sociales de empatía, respeto, transparencia, capacidad de observar, escuchar y preguntar (Wasik y Bryant, 2001) que permitan entablar una interacción y vínculo con los integrantes de la familia. Asimismo, es importante que el agente visitador tenga la sensibi- 
lidad adecuada para resguardar la privacidad de la familia y la sinceridad bajo la cual su actuar se da en un ambiente tan propio como lo es el hogar (Allen y Tracy, 2004).

\section{Método del estudio}

Desde un enfoque mixto, el método del estudio contempló la aplicación de dos técnicas de recolección de información:

1) Una encuesta a una muestra total de 51 estudiantes de cuarto y quinto año de la carrera de Trabajo Social de una universidad perteneciente al Consejo de Rectores de Universidades Chilenas $(\mathrm{CRUCH})$

2) Un grupo de discusión con 10 estudiantes de cuarto y quinto año de la carrera de Trabajo Social que con anterioridad habían contestado la encuesta.

\section{Encuesta}

El diseño de la encuesta consideró las principales variables presentes en los objetivos del estudio, las cuales fueron:

- Uso de visita domiciliaria en experiencias de práctica.

- Frecuencia del uso de la visita domiciliaria.

- Forma de participación del estudiante en la aplicación de la visita domiciliaria.

- Objetivo de la visita domiciliaria.

- Formas de coordinación de la visita domiciliaria.

- Tiempo de duración de la visita domiciliaria.

- Instrumento o material para la visita domiciliaria.

- Formas de registro y uso de éstos.

- Opinión sobre la suficiencia de la formación universitaria en la aplicación de la visita domiciliaria.

- Dificultades en la aplicación de la visita domiciliaria.

- Sentido/propósito de la visita domiciliaria.

Previo a su aplicación, la encuesta fue evaluada en dos instancias. La primera fue su aplicación piloto en cinco estudiantes de cuarto y quinto año de la carrera, quienes recibieron las indicaciones para completar la encuesta. Una vez logrado, los encuestados compartieron de forma oral y grupal sus preguntas y comentarios al instrumento, y luego registraron sus observaciones en el mismo instrumento. Esta evaluación fue un valioso espacio de retroalimentación y mejoramiento de la encuesta. La segunda instancia fue la evaluación del instrumento por parte de dos expertas académicas de la carrera de Trabajo Social de la universidad que participó del estudio. Esta vez, también se recibieron comentarios aportadores que permitieron mejorar el instrumento a aplicar.

La encuesta quedó formulada con un total de 18 ítems. Dieciséis de ellos fueron preguntas cerradas y dos de ellas abiertas. El instrumento fue precedido por la firma del formulario de consentimiento informado exigido por el Fondecyt.

Como se señaló, la muestra a la que se le aplicó el estudio correspondió a 51 estudiantes de cuarto y quinto año de la carrera de Trabajo Social. El principal criterio de selección fue que los encuestados pertenecieran a la carrera de Trabajo Social y a los dos últimos años, puesto que ello garantizaba que hubiesen tenido al menos una experiencia de práctica profesional inicial o final. La participación de los estudiantes en la encuesta fue libre y voluntaria. El procedimiento del encuestaje consideró la aplicación en aula de clases previa solicitud de autorización del docente a cargo de la asignatura.

Los resultados de la encuesta fueron ingresados a una base de datos en SPSS y fueron analizados mediante una estrategia de análisis estadístico descriptivo univariado. Respecto a las características de los encuestados, puede señalarse que del total, el $84,31 \%$ corresponde a mujeres y el $15,69 \%$ a hombres. El 90,2\% se encuentra en un rango de edad entre los 21 a 25 años y un 9,8\% en el rango "26 años o más". Los encuestados cursan cuarto o quinto año de la carrera. Un 60,78\% corresponde a estudiantes de séptimo semestre, mientras que los estudiantes de noveno semestre o quinto año de la carrera corresponden a un 35,29\%.

\section{Grupo de discusión}

Como una estrategia de recolección de información que permitiera profundizar en la opinión de los estudiantes sobre la visita domiciliaria a partir de sus experiencias, se contempló la realización de un grupo de discusión. Consistente con los objetivos del estudio, el diseño del grupo de discusión contempló las siguientes categorías:

- Definición de visita domiciliaria a partir de sus experiencias de práctica profesional.

- Limitaciones y fortalezas de la visita domiciliaria como estrategia de intervención.

- Evaluación de las experiencias de aplicación de visita domiciliaria. 
Como se mencionó, participaron un total de 10 estudiantes de cuarto y quinto año de la carrera. Dado que los participantes fueron estudiantes que previamente habían respondido la encuesta, se asumieron los mismos criterios de selección. Sin embargo, la muestra fue intencionada, puesto que fueron invitados al encuentro estudiantes de los que el equipo de investigación tenía antecedentes de haber utilizado la visita domiciliaria en sus experiencias de práctica. El procedimiento de aplicación del grupo de discusión fue invitarlos a un almuerzo y tuvo una duración de 90 minutos.

Los resultados del grupo de discusión fueron transcritos y posteriormente se realizó un análisis de contenido.

\section{Resultados del estudio}

Los resultados se organizan a partir de las principales variables y categorías presentes en los objetivos del estudio, partiendo con los resultados de la encuesta y siguiendo con los del grupo de discusión.

\section{Utilización de la visita domiciliaria según nivel de práctica}

De los estudiantes encuestados, un 64\% reportó que utilizó la visita domiciliaria mientras que un 36\% no la utilizó en su primera experiencia de práctica profesional (Figura $\mathrm{N}^{\circ} 1$ ).

FIGURA No 1 :

\section{¿APLICASTE LA VISITA DOMICILIARIA EN ESTA EXPERIENCIA DE PRÁCTICA INICIAL? (PRÁCTICA I y II)}

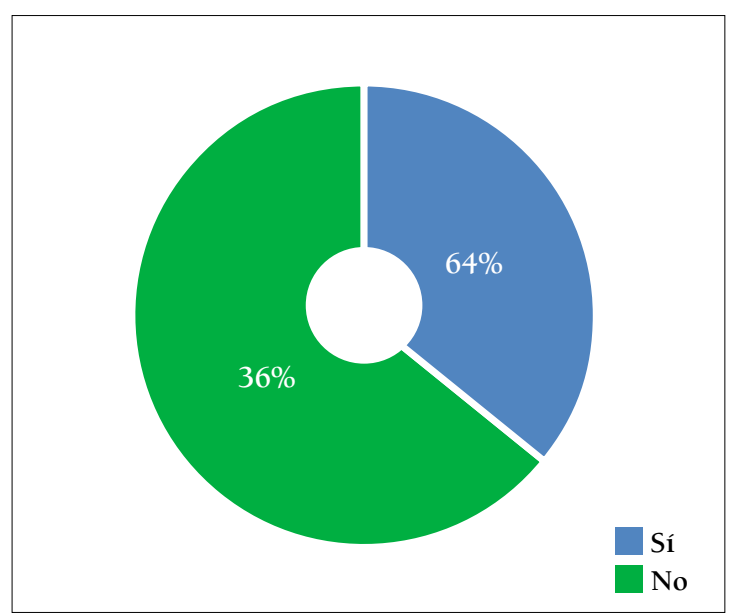

Fuente: Elaboración propia

Los encuestados revelan que en la primera práctica suelen insertarse en centros de práctica de tipo organización no gubernamental (ONG), con un $30,6 \%$. En segundo lugar, en municipalidades, con un 22,45\%, es decir, 11 estudiantes. En tercer lugar, los establecimientos educativos (escuelas o liceos) con un 14,29\%, es decir, 7 estudiantes. En cuarto lugar, en Gendarmería, con un 10,20\%, equivalente a 5 estudiantes (Figura $\mathrm{N}^{\circ} 2$ ).

FIGURA N ${ }^{\circ} 2$ :

IDENTIFICA EL TIPO DE ORGANIZACIÓN DONDE REALIZASTE O ESTÁS REALIZANDO TU PRÁCTICA: PRÁCTICA INICIAL (I y II)

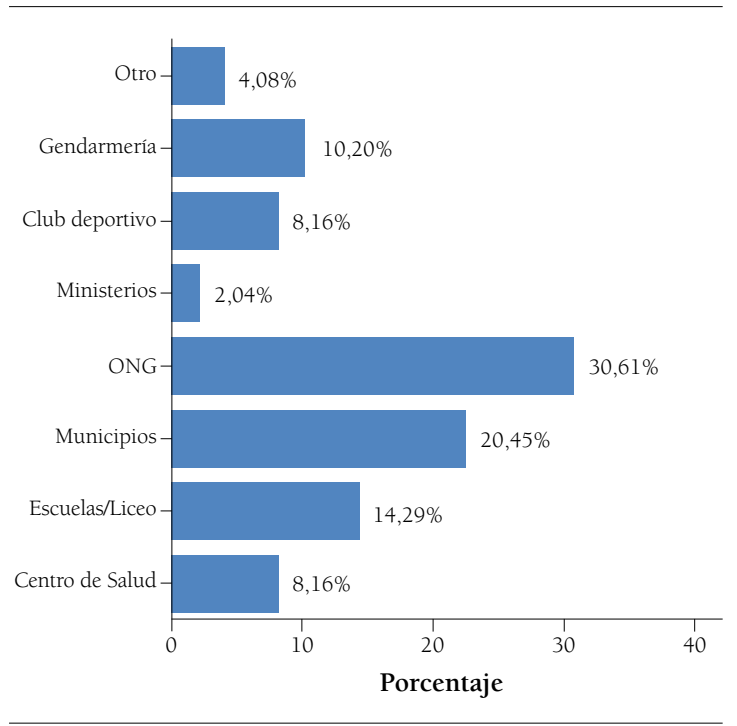

Fuente: Elaboración propia

En el caso de los estudiantes que declararon estar realizando su segunda práctica profesional, un 42,11\% señala haber utilizado la visita domiciliaria y un $57,89 \%$ declara no haberla utilizado (Figura No 3 ).

FIGURA No 3 :

\section{¿APLICASTE LA VISITA DOMICILIARIA EN ESTA EXPERIENCIA DE PRÁCTICA PROFESIONAL? (PRÁCTICA I y II)}

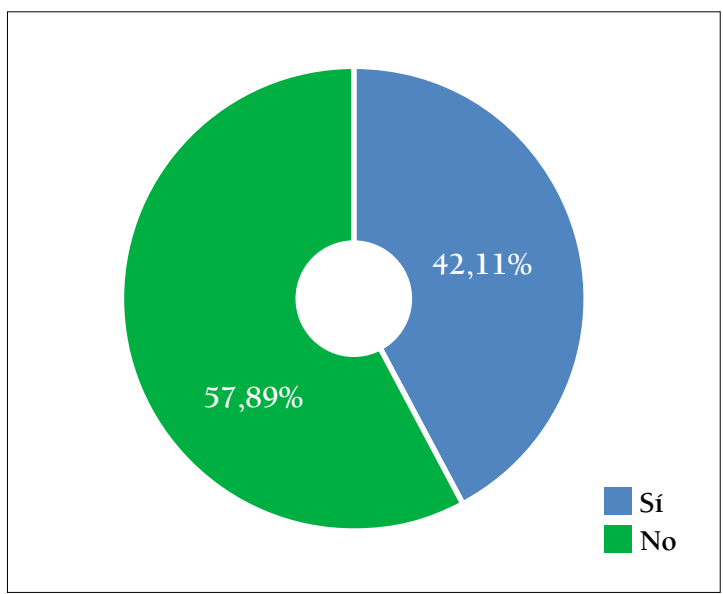

Fuente: Elaboración propia 
La principal razón reportada por los encuestados para no utilizar la visita domiciliaria fue que la estrategia no se adecuaba a los objetivos del proyecto de intervención social (60\%). Dentro de las otras razones, se explicó que el trabajador social estaba muy ocupado y no podía realizarse sin él, o que el profesional que ofició de supervisor no era trabajador social.

Los estudiantes de quinto año declararon realizar su práctica de nivel profesional mayoritariamente en organizaciones no gubernamentales $(36,8 \%)$ seguido de empresas (21,02\%). Por último, 4 estudiantes seleccionan la opción "Otro tipo de organizaciones", representando un 21,05\%, siendo estas organizaciones la "Mutual", la "Corporación municipal" y 2 estudiantes mencionan como centro de práctica un "Organismo colaborador de SENAME (PROMESI)" (Figura $\mathrm{N}^{\circ} 4$ ).

FIGURA No 4 :

IDENTIFICA EL TIPO DE ORGANIZACIÓN DONDE REALIZASTE O ESTÁS REALIZANDO TU PRÁCTICA: PRÁCTICA PROFESIONAL (I y II)

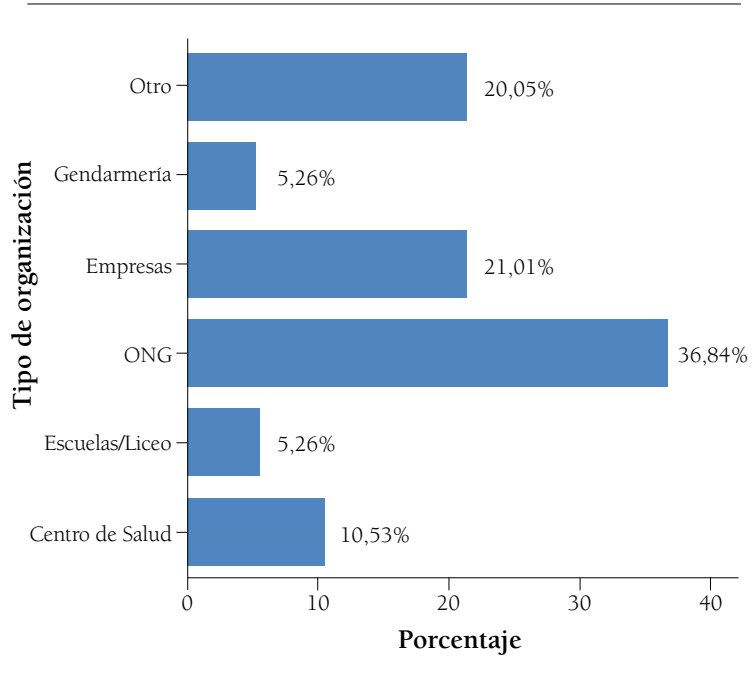

Fuente: Elaboración propia

\section{Objetivos de la visita domiciliaria}

Los encuestados que reportaron haber utilizado alguna vez esta estrategia en su(s) experiencia(s) de práctica manifiestan ocuparla principalmente con la finalidad de recolectar información del usuario para realizar diagnósticos, seguimiento o evaluación del caso (Figura $\mathrm{N}^{\circ}$ 5). Sólo 10 encuestados mencionan haberla utilizado para realizar intervención directa con el caso y su familia. Cabe señalar que este ítem de la encuesta que preguntaba sobre el objetivo de la visita domiciliaria le permitía al encuestado marcar más de una alternativa.
FIGURA N ${ }^{\circ} 5$ :

\section{MAYORITARIAMENTE, ;CUAL FUE EL OBJETIVO DE LAS VISITAS DOMICILIARIAS REALIZADAS?}

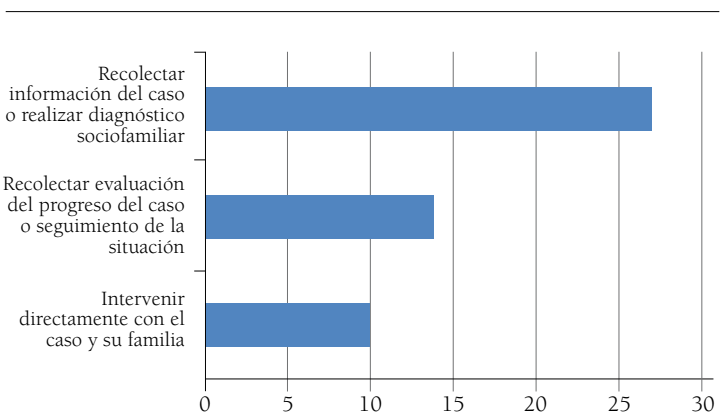

Fuente: Elaboración propia

Un $84,8 \%$ de los encuestados señalaron que el objetivo de la visita domiciliaria era siempre informado a los usuarios de la intervención (Figura $\mathrm{N}^{\circ}$ 6).

\section{FIGURA N ${ }^{\circ}$ 6: \\ ¿CON QUÉ FRECUENCIA SE INFORMABA \\ AL USUARIO EL OBJETIVO DE LA VISITA DOMICILIARIA REALIZADA?}

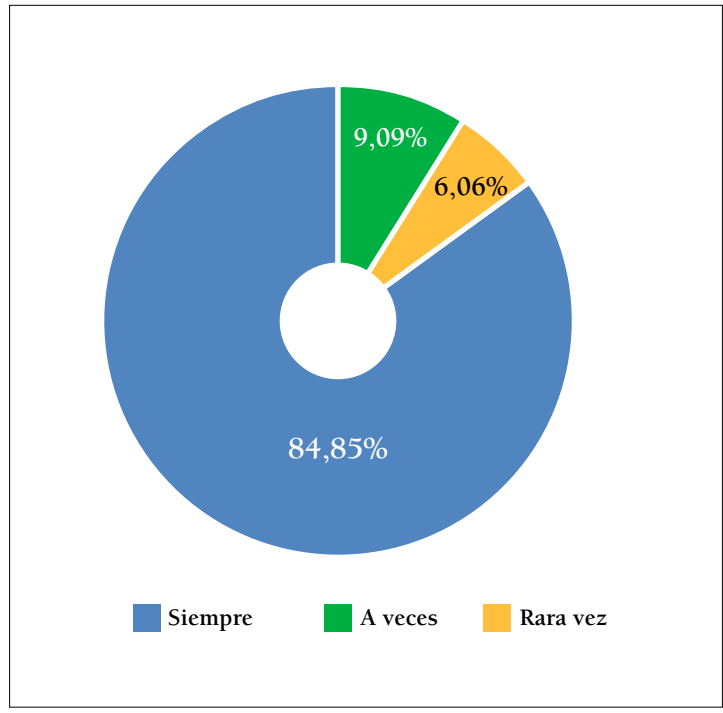

Fuente: Elaboración propia

En la mayoría de los casos, los encuestados señalan haber coordinado previamente la visita domiciliaria con el usuario (Figura No 7 ). 
FIGURA No 7 :

GENERALMENTE, ¿CÓMO SE COORDINABA LA VISITA DOMICILIARIA CON EL USUARIO?

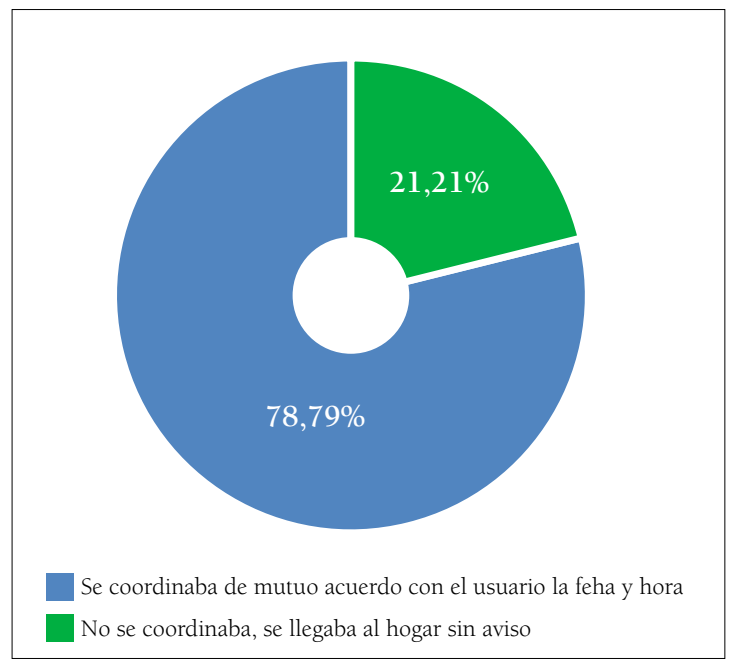

Fuente: Elaboración propia

Consistentemente con el tipo de objetivo de la visita domiciliaria, los estudiantes señalaron realizarla acompañados principalmente de un cuaderno de campo y pautas de entrevista u observaciones (Figura $\mathrm{N}^{\mathrm{o}} 8$ ). Solo 7 encuestados señalaron haber portado un material educativo o informativo a trabajar con el usuario.

\section{FIGURA N ${ }^{\circ} 8$ : \\ ¿QUÉ TIPO DE INSTRUMENTO O MATERIAL LLEVAS AL MOMENTO DE REALIZAR LA VISITA DOMICILIARIA?}

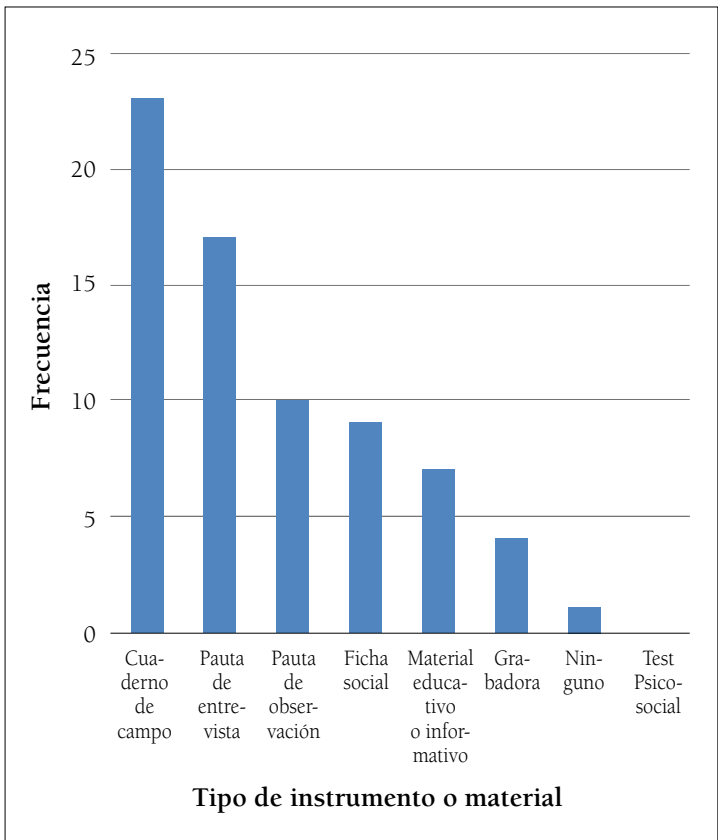

Fuente: Elaboración propia
Casi la totalidad de los encuestados (94\%) declaró haber registrado la información recolectada durante la visita domiciliaria, siendo por lo general el producto un informe social. Asimismo, casi la totalidad de los encuestados (95\%) opinó que la formación recibida en la universidad sobre visita domiciliaria era insuficiente o deficiente.

\section{Frecuencia y duración de la visita domiciliaria}

Los encuestados que declararon haber utilizado la visita domiciliaria tienden a utilizarla o con poca frecuencia (menos de 5 veces) o con mucha frecuencia (más de 21 veces) (Figura No 9).

\section{FIGURA N ${ }^{\circ}$ 9: \\ CONSIDERANDO TUS EXPERIENCIAS DE PRÁCTICA COMO ESTUDIANTE DE TRABAJO SOCIAL. ¿APRÓXIMADAMENTE, CUÁNTAS VECES TE CORRESPONDIÓ REALIZAR VISITAS DOMICILIARIAS EN TOTAL?}

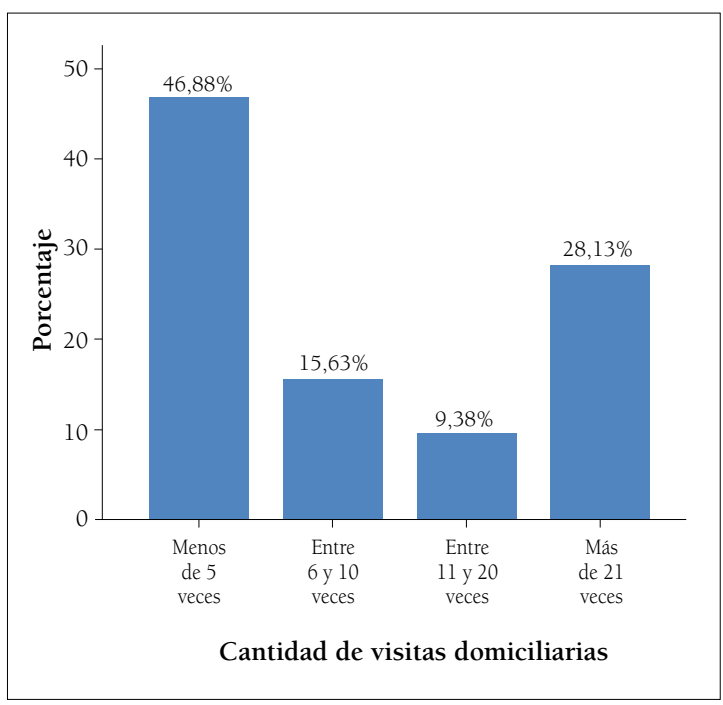

Fuente: Elaboración propia

La mitad de los encuestados señala haber realizado solo una visita domiciliaria al caso $(51,5 \%)$ o familia que estaba siendo intervenido, mientras que la otra mitad señala haberlo hecho en más de una ocasión $(48,4 \%)$. De este grupo, cerca de un $70 \%$ señala haber realizado entre 2 a 3 visitas al mismo caso. En estos casos, destaca una de las opiniones de los participantes del grupo de discusión que indican la importancia que tiene la primera visita domiciliaria que marcaría un cierto sello a como se desarrollarán en el futuro: "... es fundamental la primera visita domiciliaria que uno hace a la casa de la persona, porque ahí tú estás trazando cómo van a ser futuros encuentros y también estás trazando que a lo mejor no van a haber futuros encuentros" (E3). 
Casi la mitad de quienes realizaron visitas domiciliarias las hicieron en calidad de responsable principal y la otra mitad como acompañante o apoyo del trabajador social (Figura No 10).

FIGURA N ${ }^{\circ} 10$ :

PRINCIPALMENTE, ¿QUÉ TIPO DE PARTICIPACIÓN TUVISTE EN LAS VISITAS DOMICILIARIAS?

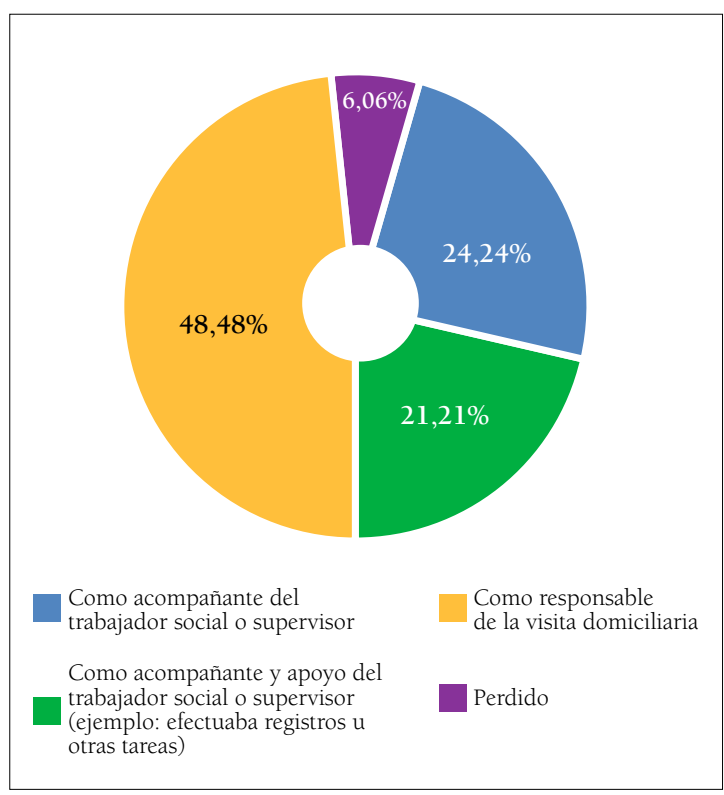

Fuente: Elaboración propia

En promedio, los encuestados señalaron realizar visitas domiciliarias de duración menor a 45 minutos (Figura $\mathrm{N}^{\mathrm{o}} 11$ ).

FIGURA $N^{\circ} 11$ :

INDIQUE EL PROMEDIO, ¿CUÁL ERA EL TIEMPO DE DURACIÓN DE LAS VISITAS DOMICILIARIAS REALIZADAS?

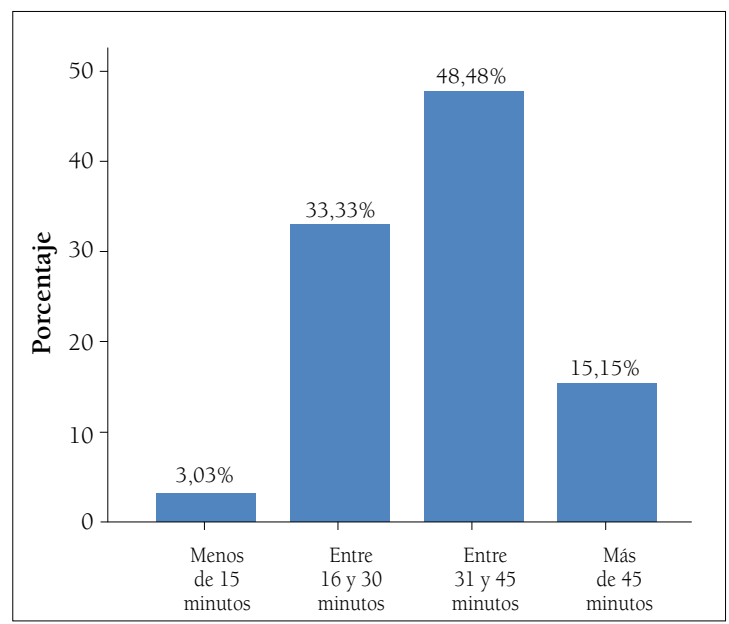

\section{Propósito o sentido de la visita domiciliaria para el estudiante de Trabajo Social ${ }^{8}$}

En primer lugar, los entrevistados conciben la visita domiciliaria como un medio, una herramienta y una técnica y no como un objetivo o fin en sí misma. Sin embargo, coinciden en apreciar que la visita domiciliaria siempre tiene que tener un objetivo y debe ser previamente planificada, es decir, no es improvisable: “... uno siempre tiene que ir con un objetivo, qué va a decir, cuáles temas no se te pueden escapar, etc. Y aunque siempre la visita es muy natural, es muy muy conversacional y todo eso, tú no puedes ir, aunque ya hayas visto a la familia no sé cuántas veces, no puedes ir sin una planificación, no puedes ir sin aunque sea anotar, no puedes olvidar que la persona tiene que renovar la visa no sé qué, no puedes olvidar de que esta persona... (E2)". Destacan que idealmente debe ser planificada en equipo para que sea eficiente: “... planificar como en equipo, porque a nosotros nos pasaba que en mi práctica habian distintos practicantes de distintas universidades; entonces, cada uno hacía su visita domiciliaria con cada grupo. Entonces, al final algunos hacían la visita domiciliaria más eficiente, porque planificaban mejor, pero otros no conseguían lo mismo" (E3). Pero no cualquier equipo, sino que deseablemente este debiese ser interdisciplinario: "que el trabajo con otros profesionales, con trabajadores sociales contribuye mucho cuando uno hace visitas domiciliarias, porque ellos te ponen en contexto con las familias, que es más allá en términos sociales, sino que también en términos del psicólogo o educador social en mi caso, dan un contexto que también que tiene que ver con el niño, que como ya decía, yo no trabajo directamente con el niño, y ellos me proveen ese contexto desde el niño" (E6).

Los estudiantes la visualizan como una herramienta que no es neutra, en el sentido que implica una desigual distribución de poder entre el profesional y el sujeto: “. . . creo que siempre se deja ver esta relación de poder, porque... sobre todo desde el área de salud, porque al final la visita domiciliaria es donde se juega si la persona se va a su casa o no, entonces hay una relación de poder en donde la persona no puede elegir si quiere que vayan a visitarla o no; la visita se hace sí o sí; entonces siento que el trabajador social o el terapeuta o quien vaya tiene un poder súper grande" (E10). También se destaca su utilidad como herramienta prácticamente única para trabajar con población reacia a participar de los programas sociales.

Consistentemente con los resultados de la encuesta, los estudiantes la conciben como un medio que sirve a procesos de indagación para conocer el contexto

Fuente: Elaboración propia

8 De aquí en adelante, el análisis es realizado a partir de los resultados del grupo de discusión. 
sociofamiliar y las dinámicas de las familias de un usuario. En la Figura 12 se resumen los principales verbos utilizados por los estudiantes para describir el propósito de la visita domiciliaria, así como también el objeto, sujeto y propósito de la estrategia. Sólo un estudiante la destaca no solamente como una herramienta útil en la etapa de diagnóstico sino que también como una estrategia de intervención directa con los sujetos: "... la utilizamos como herramienta de diagnóstico, como eh... intervención en el sentido de realizar actividades con ellos y también como una visita de cierre" (E12).

En la Figura N 12 se observan los principales verbos utilizados por los entrevistados para referirse al propósito de la visita domiciliaria. Tienen directa relación con el acto profesional de conocer y no de transformar la realidad conocida. ¿Qué refieren conocer? Los estudiantes indican principalmente que el contexto, dinámicas y problemáticas presentes no están solamente en las personas, sino que también en las familias y barrios.

\section{FIGURA N ${ }^{\circ} 12$ : \\ PROPÓSITO DE LA VISITA DOMICILIARIA DE ACUERDO A RELATO DE ESTUDIANTES PARTICI- PANTES DEL GRUPO DE DISCUSIÓN.}

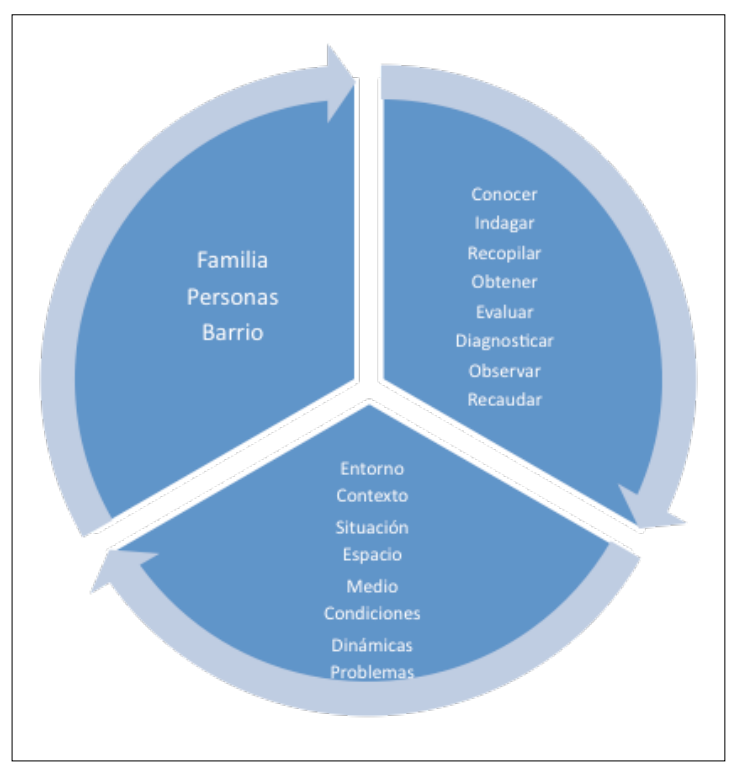

Fuente: Elaboración propia

\section{Dificultades reportadas en la utilización de la vi- sita domiciliaria}

Cinco fueron las principales dificultades que reportaron los estudiantes participantes del grupo de discusión respecto a la aplicación de la estrategia:

- Formación insuficiente en el uso de la visita domiciliaria, tanto en su dimensión teórica como metodológica. A juicio de los estudiantes, una de las consecuencias de esto es la improvisación en la aplicación de la estrategia: "(...) dentro de las limitaciones, voy a hablar súper personalmente, está el tema de la falta de preparación. O sea, a nosotros nos dijeron: ya, chiquillas, apliquen la ficha, vayan a hacer la visita domiciliaria; pero a veces uno no sabe cómo pararse frente a una persona" (E1). No se manifiesta que esta dificultad haya sido paleada con autoformación ni tampoco con alguna capacitación en sus centros de práctica.

- Planificación de la visita domiciliaria, al no contar con una pauta ni con un protocolo que permita guiar de manera más efectiva la visita domiciliaria. Los estudiantes valoran la importancia de contar con este proceso y que sea construido con el equipo de trabajo, tal como lo manifiesta el estudiante en la siguiente cita: "(...) lo que uno quiere ver es lo que ve y que no va necesariamente de la mano con lo que se espera, porque por ejemplo me ha pasado que voy a la visita, se lleva la planifcación, se hace la planificación y después estoy con mi supervisora y mi supervisora me dice 'ya, pero iy supiste o esto o viste esto?', y era como 'ah, pero es que tenía otra planificación', entonces como que de cierta forma... uno ve lo que quiere ver" (E3).

- Coordinación práctica de la visita domiciliaria, como por ejemplo dificultades en el trazado de rutas y accesibilidad de los domicilios, direcciones incorrectas, ausencia de usuarios en la vivienda, tiempo escaso de los usuarios y, por sobre todo, de la trabajadora social: “... como profesionales, la demanda es tan alta, que esta técnica puede estar limitada por términos de tiempo. Por ejemplo, en el departamento en que yo estuve, la emm, había solamente una encargada, una trabajadora social para toda la comuna. ¿A qué hora haces la visita domiciliaria; Tú vas a hacer la visita domiciliaria y la persona no está, perdiste de hacer dos visitas domiciliarias más" (E4).

- Implementación de la visita domiciliaria y desarrollo de vínculo profesional. Aquí los estudiantes destacan la prevalencia de un sentimiento de invasión o intromisión en la intimidad de un hogar, lo que traería como consecuencia la artificialidad de la situación y en especial de la conducta de los usuarios: “... uno igual siempre va a invadir un hogar, o sea siempre va a ser un tercero extraño, entonces las personas, ya sea uno les avise, acuerde una fecha o vaya de sorpresa, igual va a cambiar la... las actitudes que van a tener las personas (...) Yo creo que nunca la gente se va a comportar como naturalmente..." (E2). 
Esta situación se agudiza en un contexto de desconfianza, que se acentúa en los usuarios que están desvinculados del trabajo institucional: “... en ocasiones provoca desconfianza dentro de las personas, em... me imagino con las personas que principalmente están muy desvinculadas de las instituciones emm... que nunca acuden, que se yo no sé a una comuna, a la municipalidad (...) no tienen idea por qué se los está visitando, no tienen idea por qué tienen que abrir la puerta a alguien..." (E6).

- Seguridad y autocuidado profesional. En este ámbito, los estudiantes expresan enfrentar riesgos por el contexto del territorio a intervenir, como también refieren poco manejo para actuar en situaciones hostiles, imprevistas o contingencias propias de la vida cotidiana de una familia. En este sentido, los recursos vinculados al desplazamiento son un aspecto importante para minimizar los riesgos y son una variante del tipo de organización en la cual el estudiantes se inserta: "... va a depender mucho de la institución en la que se esté, porque creo que muchos de acá tienen que ir en micro y tomar riesgos de repente. En cambio, hay otros que tienen disponibilidad de recursos y que prestan móviles. Entonces, yo creo que es súper importante el tema de los recursos en las visitas domiciliarias, es súper importante ese aspecto y es algo que hay que ver" (E5).

Por otra parte, la alta complejidad social y familiar de las temáticas que suelen ser abordadas por los trabajadores sociales, suponen una alta carga emocional que en un contexto de poca experiencia profesional puede ser aún más complejo de manejar, tal como se refleja en la siguiente cita: “... yo estoy en mi práctica de emm explotación sexual comercial infantil, ehh visitar a las familias, que los adultos, yo no trabajo con los niños, yo trabajo con los adultos responsables de esos niños, las familias principalmente, pero es ... ellos de por sí tienen una carga emocional alta, temas de culpabilidad, temas que también te traspasan a ti y que también generan emoción y, como decía, si no te genera, si no te pasa nada, no es muy bueno. Eso también uno le llega, uno queda con eso, uno no puede estar haciendo otras visitas. Y que un trabajo que donde lo dejai después del día de práctica. Te vai a tu casa y donde queda esa carga emocional" (E5). Esto refleja escaso manejo de estrategias de autocuidado profesional.

\section{Fortalezas reportadas por los estudiantes sobre la visita domiciliaria}

Las fortalezas que los participantes del grupo de discusión le asignan a la visita domiciliaria tienen relación con sus ventajas, las que fueron las siguientes:

- Posibilita construir relaciones cercanas con las personas en el medio en el cual cotidianamente viven, tal como lo señala este entrevistado: “... dentro de la práctica profesional yo creo ha sido una de las herramientas más potentes que tengo como para acercarme a las familias..." (E11). Relaciones que permean el trabajo profesional y generar cambios en la relación de ayuda: “... logramos entablar una relación con la persona, poder a partir de sus propias creencias lograr darle un giro a lo que nosotros nos enseñaron, porque nosotros íbamos a llevarle la teoría (...) pero al momento de entablar una relación con él, pudimos ver sus intereses, ver otras posibles herramientas en las que lo podíamos ayudar y así lograr trabajar de mejor manera esta instancia que teníamos que era la visita domiciliaria" (E2).

- Permite comprender las dinámicas de las familias y la situación de cada una de acuerdo a su propio contexto: “... que uno puede ver las dinámicas positivas o negativas de las familias, que a lo mejor, avisándose o no avisándose, en algún momento va a decir la información, va a salir la pelea o va a salir las cosas negativas o positivas de la familia" (E7).

- Facilita el acceso a personas que presentan algún tipo de discapacidad o dificultad de desplazamiento, como lo constata el entrevistado: “... es una fortaleza importante el poder acceder a personas que tienen problemas, tanto de tiempo, físico, qué sé yo, como es en el caso de personas con discapacidad, que no pueden ir fácilmente a la institución que sea, es una gran fortaleza, una gran fortaleza de las visitas" (E8).

\section{Cuidados profesionales éticos en la aplicación de la visita domiciliaria}

Los estudiantes no fueron indiferentes a los problemas éticos que pueden producirse por el uso de esta estrategia. El principal consenso que hubo entre ellos es que una visita domiciliaria mal realizada podría ocasionar daño en los sujetos, razón por la cual es parte de la ética del profesional desarrollar efectivamente el proceso de visita domiciliaria, siendo conscientes que no es una relación igualitaria, como se aprecia en el siguiente relato: “... la visita domiciliaria es un arma de doble filo, porque igual es un instrumento de poder en donde uno se mete dentro de la familia, y que muchas veces los trabajadores sociales no tienen o no tenemos las habilidades desarrolladas para realizar una visita domiciliaria. Donde muchas veces se aplican esta asimetría para sacar información, enton- 
ces, la visita domiciliaria es súper positiva desde cierto lado, pero que también mal utilizada puede ser un arma súper prejuiciosa y que al final te conlleva a romper el vinculo con las personas y con el grupo" (E10).

En el tema ético también hubo desacuerdos y debate entre los integrantes del grupo de discusión. Uno de los asuntos éticos que fue objeto de disensos fue si era necesario avisar o no, como se revela en el siguiente extracto de entrevista: “... dependiendo del carácter que tenga tu visita, dependiendo del fenómeno de intervención que tú tienes, puede ser avisada o no ser avisada. Por ejemplo, en el caso de negligencia parental, una pareja que tiene la escoba en la casa, yo no avisaría porque tú tienes que dar cuenta de la realidad que hay, porque si es una persona que qué sé yo, tiene la tendalá en la casa y tú, tú, recibes la denuncia de vecinos, qué sé yo, de hay maltrato familiar, claro, tú no puedes, tú no puedes... es... yo creo que sería efectivo por el bien mayor de los niños, qué sé yo, el no avisar, para ver las dinámicas como corresponden..." (E9). Respecto de este asunto, aproximadamente dos tercios de los estudiantes expresaron que era necesario siempre avisar, mientras que un tercio flexibilizaba ese criterio si es que el problema que ocasionaba la visita era por alguna vulneración de derecho de los niños.

\section{Discusión de resultados}

Un porcentaje no menor de estudiantes declararon haber utilizado la visita domiciliaria en sus distintas experiencias de práctica profesional, especialmente en la primera práctica, lo que refleja que es una estrategia que sigue siendo vigente para el Trabajo Social. En este sentido, se concuerda con lo establecido por Tonon (2005) y González (2014) respecto al papel estratégico que tiene para la disciplina. Sin embargo, al momento de profundizar en la forma en la cual es utilizada, se observan ciertas brechas respecto del uso declarado por los estudiantes y lo que los estudios revisados sugieren realizar. Por ejemplo, respecto a la frecuencia, la aplicación de la estrategia a cada caso o familia es mínima (la mitad reportó haberla utilizado solo 1 vez para el caso o familia) comparado con lo que los estudios revisados indican para que sea una técnica efectiva que, por ejemplo, se refieren a tres veces al mes como mínimo (Nievar et al., 2010, y Tubach et al., 2012). Otro de los aspectos encontrados en el presente estudio que difiere de otros es el tiempo de duración de las visitas, puesto que todos los estudiantes reportaron haber realizado visita de menos de 45 minutos, siendo que, por ejemplo, el sistema Chile Crece Contigo (2009) sugiere que el agente visitador permanezca en el hogar al menos 60 minutos.
Un hallazgo de especial relevancia para este estudio consiste en que se detectó que en la muestra de estudiantes la visita domiciliaria se utiliza principalmente con propósitos de recolección de información para efectos de diagnóstico o seguimiento y no como estrategia para realizar una entrega efectiva de servicios educativos, de ayuda o apoyo concreto, como lo sugiere el estudio de Aracena et al (2011) y el mismo sistema Chile Crece Contigo. Eso también se comprueba al momento de ver los resultados al ítem de los instrumentos o materiales que acompañan la visita domiciliaria, en el que la mayoría reporta llevar cuaderno de campo, pauta de entrevista, de observaciones y ficha social, pero pocos reportan llevar algún material educativo o informativo. Esto refleja una posible subutilización de la estrategia en los distintos contextos de intervención en los que se introducen los estudiantes.

Si se considera que la preparación de la visita y la capacitación del visitador resultan fundamentales para la efectividad de la estrategia (Tubach et al., 2012), entonces es preocupante que los estudiantes de la muestra hayan referido como una de las dificultades sentirse poco preparados para aplicarla tanto desde el punto de vista teórico como metodológico.

En sintonía con lo propuesto por Niever et al. (2010), Allen y Tracy (2004), Niever et al. (2011), Lazaro et al. (2007) y Aylwin y Solar (2011), las fortalezas de la visita domiciliaria son también valoradas por los participantes del estudio, en el sentido que permite apreciar y conocer mejor la situación de las personas y las dinámicas de la familia, en el medio natural, y también acceder a personas que presentan algún tipo de barrera para acceder a los servicios institucionales que se realizan en dependencias institucionales.

Resulta de especial interés el desacuerdo percibido durante el grupo de discusión por los estudiantes respecto a un aspecto ético relevante, como lo es si corresponde o no avisar de la realización de la visita en un contexto de vulneración de derechos de los niños. En este sentido, es importante que el agente visitador actúe con sinceridad (Allen \& Tracy, 2004) y establezca un acuerdo previo con los adultos durante la realización de la visita, donde se determine en conjunto quién realizará la visita, lugar, día, hora, quienés participarán y objetivos o temas que serán abordados (Chile Crece Contigo, 2009). Esto puede resultar clave en la efectividad de la estrategia. 


\section{Conclusiones}

Como una manera de aportar al estudio empírico de una de las estrategias de intervención más tradicionales del Trabajo Social en Chile, se llevó a cabo esta investigación que se abocó a describir cómo valoran y utilizan la visita domiciliaria un grupo de estudiantes de Trabajo Social de una universidad del CRUCH que ha tenido alguna experiencia de práctica profesional. Gracias a este estudio, se pudo constatar que, en este grupo de estudiantes, la estrategia es utilizada y bien valorada por sus aportes. No obstante, su aplicación no está exenta de dificultades y desafíos. En este sentido, uno de los principales desafíos es promover el tránsito desde un uso de la visita para fines de recolección de información a uno como estrategia de actuación profesional para construir una relación de trabajo con el usuario y generar los cambios anhelados por el proceso de intervención social. Otro de los desafíos que se trazan desde este estudio es la importancia de incorporar con mayor fuerza dentro de los currículum de formación de trabajadores sociales a la visita domiciliaria como estrategia de intervención social.

\section{Referencias bibliográficas}

ALLEN. S. \& TRACY, E. (2004). Revitalizing the role of home visiting by school social workers. Children \& Schools, vol.26, n.4, 197-207.

ARACENA, M., LEIVA, L., UNDURRAGA, C., KRAUSE, M., PEREZ, C., CUADRA, V., CAMPOS M. Y VEDREGAL, P. (2011). Evaluación de la efectividad de programas de visitas domiciliarias para madres adolescentes y sus hijos/as. Revista Médica de Chile, v.139, n. $1,60-65$.

ASCENCIO-HUERTAS, L., RANGEL-DOMÍNGUEZ, N. Y ALLENDE, S. (2013). Proceso de atención psicológica en el programa de atención y cuidados en oncología del Instituto Nacional de Cancerología. Psicooncología, vol.10, n.2-3, 393-406.

ASTUDILlO, W., MENDINUETA, C. Y GRANJA, P. (2008). Cómo apoyar al cuidador de un enfermo en el final de la vida. Psicooncología, vol.5, n. 2-3, 359-381.

AVELLAR, S. \& SUPPLEE, L. (2013). Effectiveness of home visiting in improving child health and reducing child maltreatment. Pediatrics, vol.132, n.2, 90-99.

AYLWIN, N. Y SOLAR, M. (2011). Trabajo social familiar. Santiago: Ediciones Universidad Católica.

BAQUI, A., WILlIAMS, E., ROSECRANS, A. AGRAWAL, P. AHMED, S., DARMSTADT, G., KUMAR, V., KIRAN, U., PANWAR, D., AHUJA, R. \& SRIVASTAVA, V. (2008). Impact of an integrated nutrition and health programme on neonatal mortality in rural northern India. Bulletin of the World Health Organization, vol.86, N.10, 796-805.

BOLLER, K., STRONG, D. \& DARO, D. (2010). Home visiting: looking back and moving forward. Zero to Three. v30 n6 p4-9.

BRUGEROLLES, M., DOIS, A. Y MENA, C. (2008). Beneficio de la visita domiciliaria para los adultos mayores. Horizonte de enfermería, vol.1, n.2, 55-64.

CRUZ, M y MARTHE, M. (2010). A visita domiciliária na estratégia de saúde da família: conhecendo as percepções das famílias. Saúde Soc. São Paulo, v.19, n.3, 605-613

EPA (2005). Implementing an asthma home visit program. http://www.epa.gov/asthma/homevisits.html.

GARCÍA, C., MCNAUGHTON D, RADOSEVICH DM, BRANDT J, MONSEN K. (2013). Family home visiting outcomes for Latina mothers with and without mental health problems. Public Health Nurs, vol. 30, n.5, 42938. doi: 10.1111/phn.12054.

GOGIAA, S. \& SACHDEVA, H. (2010). Home visits by community health workers to prevent neonatal deaths in developing countries: a systematic review. Bull World Health Organ, vol. 88, n. 9, 658-666.

GONZÁLEZ, V. (2014). Cambios familiares contemporáneos: el reflejo de las relaciones familiares en el espacio doméstico y su conocimiento a través de la visita domiciliaria en el trabajo social. Revista Electronica de Trabajo Social. Edicion Online. Departamento de Trabajo Social, Facultad de Ciencias Sociales, Universidad de Concepción. http://www.trabajosocialudec.cl/ rets/2014/01/02/cambios-familiares-contemporaneosel-reflejo-de-las-relaciones-familiares-en-el-espaciodomestico-y-su-conocimiento-a-traves-de-la-visitadomiciliaria-en-el-trabajo-social/

ISSEL, L., FORRESTAL, S., SLAUGHTER, J., WIENCROT, A. \& HANDLER, A. (2011). A review of prenatal home-visiting effectiveness for improving birth outcomes. JOGNN, 40, 157- 165.

KITZMAN, H., OLDS, D., HENDERSON, C., HANKS, C; COLE, R., TATELBAUM, R., MCCONNOCHIE, K., SIDORA, K., LUCKEY, D., SHAVER, D., ENGELHARDT, K., JAMES, D. \& BARNARD, K. (1997). Visitation by nurses on pregnancy outcomes, childhood injuries, and repeated childbearing A randomized controlled trial. JAMA, vol. 278, n.88, 644-652.

KOTLIARENCO, M., GÓMEZ, E., MUÑOZ, M. Y ARACENA, M. (2010). Características, efectividad y desafíos de la visita domiciliaria en programas de intervención temprana. Revista de salud pública. 12 (2): 184-196.

LÁZARO, SANTA. (2007) Aprendiendo la práctica del Trabajo Social. Madrid: Universidad Pontificia Comillas.

LÓPEZ, L. (1949). El servicio social y sus proyecciones en la industria. Tesis (Asistente Social)--Universidad de Chile. 
MASTEN, A. \& COATSWORTH, J. (1998). The development of competence in favorable and unfavorable environments. American Psychologist. 53, 205-220.

MEDINA, M., FERNÁNDEZ, J., FUENTES, M., HERNÁNDEZ, M. Y PÉREZ, R. (1998). Evaluación del impacto en cuidadores de usuarios del Servicio de Ayuda a Domicilio. Anales de psicología, vol. 14, n. 1, 105-126.

MINISTERIO DE SALUD (2008). En el camino a centro de salud familiar. http://buenaspracticasaps.cl/ wp-content/uploads/2014/07/MINSAL-2008-En-elcamino-a-Centro-de-Salud-Familiar.pdf

NIEVAR, M., VAN EGEREN, L. \& POLLARD, S. (2010). A meta-analysis of home visiting programs: moderators of improvements in maternal behavior. Infant mental health journal, Vol. 31(5), 499-520. DOI: 10.1002/ imhj.20269.

SAI"AS T, LERNER E, GREACEN T, SIMON-VERNIER E. \& EMER A. (2012) Evaluating Fidelity in Home-Visiting Programs: a Qualitative Analysis of 1058 Home Visit Case Notes from 105 Families. PLoS ONE 7(5): e36915. doi:10.1371/journal.pone.0036915.

SISTEMA CHILE CRECE CONTIGO (2009). Orientaciones Técnicas Visita Domiciliaria Integral para el Desarrollo Biopsicosocial de la Infancia. http://www. crececontigo.gob.cl/wp-content/uploads/2010/01/ Visita-Domiciliaria-Integral-para-el-Desarrollo-Biopsicosocial-de-la-Infancia-2009.pdf.

TARDEL, G. (1947). Patronatos católicos en Santiago y sus obras sociales. Tesis (Asistente Social)--Escuela de Servicio Social Elvira Matte de Cruchaga.

TONON, G. (2005). Las técnicas de actuación profesional del trabajo social. Buenos Aires: Espacio.

TUBACH, F., GREACEN, T., SAIAS, T., DUGRAVIER, R., GUEDENEY, N., RAVAUD, P., TERENO, SUSANA, TREMBLAY, R., FLISSARD, B., GUEDENEY, A., \& CAPEDP STUDY GROUP. (2012). A home-visiting intervention targeting determinants of infant mental health: the study protocol for the CAPEDP randomized controlled trial in France. BMC Public Health. 12:648.

WASIK, B. \& BRYANT. D (2001). Home visiting. Procedures for helping families. Thousand Oaks, CA: Sage.

ZAMORANO, L. (1935). El servicio social escolar. Tesis (Asistente Social)-Pontificia Universidad Católica de Chile. 